\title{
Analysis of SEMA3B methylation and expression patterns in gastric cancer tissue and cell lines
}

\author{
RENPIN CHEN $^{1}$, XIAOJU ZHUGE ${ }^{1}$, ZHIMING HUANG $^{1}$, DEYI LU $^{1}$, XIAOHUA YE $^{2}$, \\ CHAO CHEN $^{1}$, JIEYU YU ${ }^{1}$ and GUANGRONG LU ${ }^{3}$ \\ ${ }^{1}$ Department of Gastroenterology and Hepatology, The First Affiliated Hospital of Wenzhou \\ Medical University, Wenzhou, Zhejiang 325035; ${ }^{2}$ Department of Gastroenterology, Jinhua Municipal \\ Central Hospital, Jinhua, Zhejiang 321000; ${ }^{3}$ Department of Gastroenterology and Hepatology, The Second \\ Affiliated Hospital of Wenzhou Medical University, Wenzhou, Zhejiang 325000, P.R. China
}

Received November 3, 2013; Accepted December 19, 2013

DOI: $10.3892 /$ or.2014.2972

\begin{abstract}
The family of semaphorins has been demonstrated to possess tumor suppressor activity, in which semaphorin 3B (SEMA3B) is differentially expressed in several types of tumors. The relationship between SEMA3B expression and its clinical significance in gastric cancer (GC) is currently unclear. In the present study, the expression and methylation status of the SEMA3B gene were detected by quantitative PCR and bisulfite sequencing PCR (BSP). Data indicated that the levels of SEMA3B mRNA decreased in gastric tumor tissues and the methylation status of SEMA3B in the tumor group was higher than the paired normal tissues. By BSP, the SEMA3B gene showed high methylated status which was detected in all 4 cell lines (AGS, BGC-823, MGC-803 and SGC-7901). Treatment of the cells with 5-Aza-2'-deoxycytidine revealed clearly elevated mRNA levels of SEMA3B. These results were further confirmed by western blot analysis of Sema3b protein expression. At the same time, increased expression of p53 mRNA in BGC-823, MGC-803 was detected and indicated that p53 may be involved in the regulation of SEMA3B expression in specific genetic background. Downregulation of SEMA3B was negatively correlated with tumor size and $\mathrm{N}$ staging in $\mathrm{GC}$ $(\mathrm{P}<0.05)$. In conclusion, $\mathrm{CpG}$ methylation of SEMA3B epigenetically regulates SEMA3B expression during development of GC. Furthermore, 5-Aza-2'-deoxycytidine could reverse the hypermethylation status of SEMA3B, which may benefit future studies exploring the application of demethylating agents in clinical usage of GC.
\end{abstract}

Correspondence to: Professor Zhiming Huang, Department of Gastroenterology and Hepatology, The First Affiliated Hospital of Wenzhou Medical University, Ouhai, Wenzhou, Zhejiang 325035, P.R. China

E-mail:wzhzm123@gmail.com

Key words: SEMA3B, gastric cancer, 5-Aza-2'-deoxycytidine, DNA methylation

\section{Introduction}

Gastric cancer (GC) is the 4th most common cancer and the 2nd leading cause of cancer-related mortality worldwide (1). Although treatment measures are being developed, the prognosis of GC remains poor and is correlated with tumor invasion (2). Development and oncogenesis of GC remains controversial and involves diverse factors, due to complex genetic backgrounds. Semaphorins are a large family of membrane-bound and cytoplasmic proteins, with up to 20 members (3). Semaphorins were originally identified to play a role in axonal guidance (4-7). However, further evidence showed that members of the semaphorins family also regulate cell migration, cell differentiation, growth and angiogenesis in a variety of tissues (8).

Semaphorin 3B (SEMA3B) is a member of the semaphorin family, located on 3p21.3. SEMA3B was identified as a tumor suppressor gene that is inactivated in lung cancer $(9,10)$, HEY ovarian carcinoma (11), hepatocellular carcinomas and cholangiocarcinomas (12). Multiple mechanisms are involved in the tumor suppressive role of SEMA3B that result in a two-hit theory, i.e., silencing of SEMA3B through epigenetic changes and allelic loss is a common and important event in its tumorigenesis procedure. Furthermore, various regulatory factors participate in the expression pattern of SEMA3B. Research shows that the Sema3b protein may play a role in regulating cell growth and SEMA3B has been identified as a mediator of p53 tumor-suppressor (13). SEMA3B is a potential tumor suppressor that induces apoptosis in SEMA3B-inactivated tumor cells through the Np-1 Receptor through inactivating the Akt signaling pathway (14). As a carcinogenic factor, vascular endothelial growth factor (such as VEGF165) acts as an autocrine survival factor that could block SEMA3B-mediated tumor-suppressing effects in tumor cells (15). Koyama et al (16) demonstrated that insulin-like growth factor-binding protein-6 (IGFBP-6) is the effector of tumor suppressor activity of SEMA3B. However, upregulation of furin-like pro-protein convertases in malignant cells may enable tumors to evade the antiangiogenic effects of Sema3b protein (17). SEMA3B also exerts unexpected functions in cancer progression by fostering a prometastatic environment 
through elevated IL-8 secretion and recruitment of macrophages coupled to suppress tumor growth (18).

In the present study, we characterized the gene expression and clinical meaning of SEMA3B in GC. Differential GC cell lines and tissues from gastric tumor were used for the exploration of the relationship between methylation status and expression of the SEMA3B gene. Our findings indicated that the SEMA3B gene was expressed low in GC and contributed to the growth of tumor. The demethylation effect of 5-Aza-2'deoxycytidine restored the expression of SEMA3B. We suggest that the antitumor effect of SEMA3B could be used as a new approach in the diagnosis and treatment of GC.

\section{Materials and methods}

Patients and tissues samples. Thirty patients with primary GC (22 males, 8 females; median age, 64.5 years; range, 42-80 years) undergoing gastrectomy (Billroth II or Radical Gastrectomy) between 2011 and 2013 at the First Affiliated Hospital of Wenzhou Medical University were included in the present retrospective study. Tumor samples were compared with normal gastric mucosa specimens (obtained from negative resection margin) of the same individuals. No patient received preoperative or adjuvant radio- or chemotherapy. TNM staging of tumor was performed according to the UICC/ AJCC (2010) criteria. Specimens were snap frozen in liquid nitrogen within $30 \mathrm{~min}$ after the resection for storage.

All patients were informed of the special examination of tumor samples in accordance with the ethical standards of the Committee on Human Experimentation of the First Affiliated Hospital of Wenzhou Medical University.

Cell culture and 5-Aza-2'-deoxycytidine treatment. Human GC cell lines BGC-823, MGC-803 and SGC-7901 were grown in RPMI-1640 plus 10\% fetal bovine serum (FBS; Invitrogen, Cergy Pontoise, France) at $37^{\circ} \mathrm{C}$ and $5 \% \mathrm{CO}_{2}$. The cell line AGS was grown in F12K plus $10 \%$ FBS. Cells were seeded at a density of $5 \times 10^{5}$ cells $/ 10-\mathrm{cm}$ plate on the first day. 5-Aza-2'deoxycytidine (Sigma, St. Louis, MO, USA) was added at the final concentration of 0,2 and $10 \mu \mathrm{M}$ after 10 -h starvation. Cells were harvested on days 3 and 5 for analysis of SEMA3B mRNA, protein expression and methylation status.

RNA extraction and quantitative real-time reverse transcription PCR ( $q R T-P C R)$ analysis. Total RNA was isolated from cultured cells with TRIzol reagent (Invitrogen Life Technologies, Grand Island, NY, USA) following the manufacturer's protocol and mRNA levels of SEMA3B, p53 and GAPDH were evaluated with the real-time PCR system by Bio-Rad (Bio-Rad CFX96; Bio-Rad Laboratories Hercules, CA, USA) using cDNA reverse transcription kit (High Capacity type; Applied Biosystems) and RNA-direct ${ }^{\mathrm{TM}}$ SYBR $^{\circledR}$ Green Real-time PCR Master Mix (Toyobo). Melting curves were generated for each real-time PCR to verify the specificity of each PCR reaction. An independent experiment was performed 3 times of real-time PCR for accuracy. The GAPDH gene was used as a reference gene. The $2^{-\Delta \Delta C T}$ method was used to calculate the relative cDNA amounts between different cell lines and this application was based on assumptions stated by Livak and Schmittgen (19). All primer sequences and PCR conditions used for mRNA expression and methylation analysis are shown in Table I.

Western blot analysis. Total protein was obtained from cell culture by cell lysis buffer (Beyotime Institute of Biotechnology, Shanghai, China). Then, a small volume (50 $\mu \mathrm{l})$ of lysate was removed to perform a protein assay. To the remaining volume of cell lysate, we added an equal volume of $2 \mathrm{X}$ Laemmli sample buffer. Each cell lysate was boiled in sample buffer at $100^{\circ} \mathrm{C}$ for $5 \mathrm{~min}$ and aliquoted. Lysates were stored at $-20^{\circ} \mathrm{C}$ and equal amounts of protein were loaded into the wells of the SDS-PAGE gel, along with molecular weight markers. After transferring the protein from the gel to the PVDF membrane, the membrane was blocked overnight at $4^{\circ} \mathrm{C}$ using $5 \%$ blocking solution. We respectively incubated the membranes with SEMA3B antibody (50 kDa, $1 \mu \mathrm{g} / \mathrm{ml}$; Abcam, Cambridge, MA, USA) and $\beta$-actin antibody (42 kDa; Beyotime Institute of Biotechnology) in $2 \%$ blocking solution overnight at $4^{\circ} \mathrm{C}$. After washing the membrane with TBST, horseradish-peroxidase (HRP) was added for signal development. Images were acquired using darkroom development techniques for chemiluminescence by ECL Plus (Amersham Biosciences).

Genomic DNA isolation, sodium bisulfite modification and bisulfite sequencing PCR. Genomic DNA was obtained from GC cell lines and paired specimens of normal/tumor gastric tissues. Bisulfite modification was performed by EZ DNA Methylation-Gold kit (Zymo Research, Irvine, CA, USA) based on the manufacturer's protocol. The treated DNA was amplified by PCR using the following primer with product size of 249: F, 5'-GGATTTTTTGTAGTTGGTGGAGT-3' and R, 5'-GAGAGAGGAGTTAGATAGATTTTGA-3' (Table I). Amplified PCR products were analyzed by electrophoresis on a $2 \%$ agarose gel and were purified with a TIANgel Midi Purification kit (Tiangen Biotech, Co., Ltd., Beijing, China). The PCR fragments were ligated to pMD 19-T Vector (Takara Biotechnology, Dalian, China) and transformed into E. coli DH5 $\alpha$ Electro-Cells (Takara Biotechnology). Ten subcloned colonies were chosen at random from each gastric cell line and 15 paired gastric tumor and normal tissues. PCR products were sequenced by the $3730 x 1$ DNA Analyzer (Applied Biosystems, Foster City, CA, USA).

Statistical and bioinformatic analysis. Statistical significance of two groups was determined by Student's t-test using Stata/SE (Version 11.0; StataCorp, College Station, TX, USA). The difference of DNA methylation in paired specimens was analyzed by the $\chi^{2}$ test. The correlation between the DNA methylation level and mRNA expression fold was determined by Spearman correlation analysis. The $2^{-\triangle \Delta C T}$ method was adopted to quantify the mRNA expression level between the tumor group and the normal tissue group, where $\Delta \Delta \mathrm{C}_{\mathrm{T}}$ was defined by the following equation: $\Delta \Delta \mathrm{C}_{\mathrm{T}}=\left[\left(\mathrm{C}_{\mathrm{T}}\right.\right.$ of SEMA3B $-\mathrm{C}_{\mathrm{T}}$ of GAPDH $)_{\text {Treatment }}-\left(\mathrm{C}_{\mathrm{T}}\right.$ of SEMA3B $-\mathrm{C}_{\mathrm{T}}$ of GAPDH $)$ Control]. Network relationships between SEMA3B and its correlated regulators were analyzed by Agilent Literature Search Software (20), which is a meta-search tool for automatically querying multiple text-based search engines (both public and proprietary) in order to extract associations among genes/ proteins of interest. All P-values were based on two-sided 
Table I. Primers used for SEMA3B mRNA and methylation analysis.

\begin{tabular}{llcc}
\hline Gene (method) & \multicolumn{1}{c}{ Primer sequence $\left(5^{\prime}-3^{\prime}\right)$} & Annealing temperature $\left({ }^{\circ} \mathrm{C}\right)$ & Product size $(\mathrm{bp})$ \\
\hline SEMA3B (qRT) & F: CCAGTGCCAAGAGGCGGTTC & 62 & 214 \\
& R: AGCACCTGGGTGTGGGCTGT & & \\
GAPDH (qRT) & F: GACTCATGACCACAGTCCATGC & 62 & 2113 \\
p53 (qRT) & R: AGAGGCAGGGATGATGTTCTG & 60 & 249 \\
SEMA3B (BSP) & F: GGAGCCGCAGTCAGATCCTAG & & \\
& F: CAAGGGGGACAGAACGTTG & 60 & \\
\hline
\end{tabular}

Bp, base pair; qRT, quantitative PCR; F, forward primer; R, reverse primer.

A
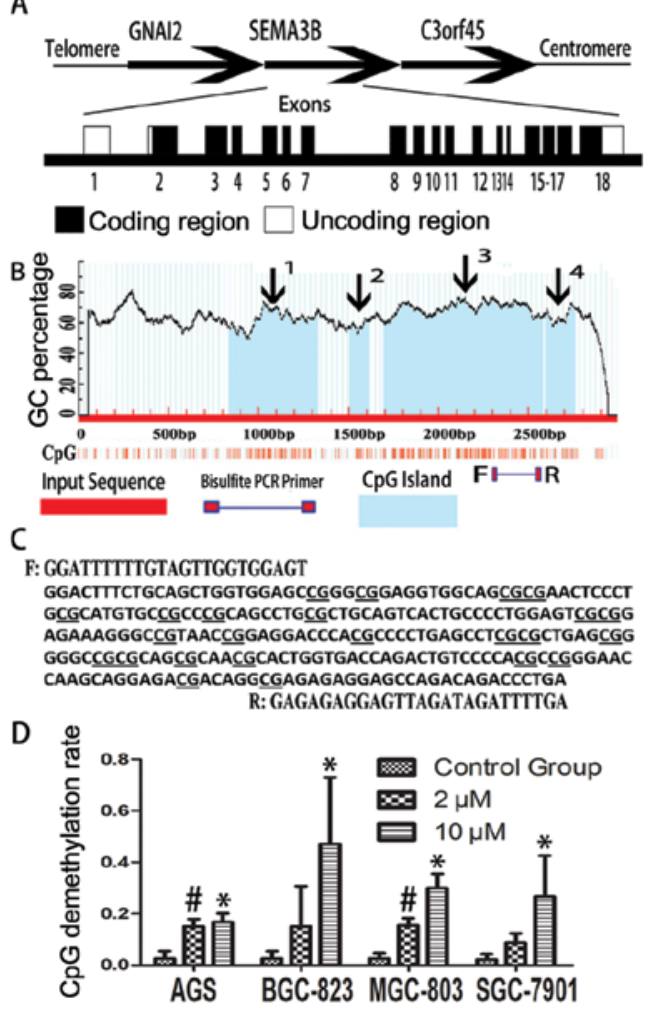

$\mathrm{E}$

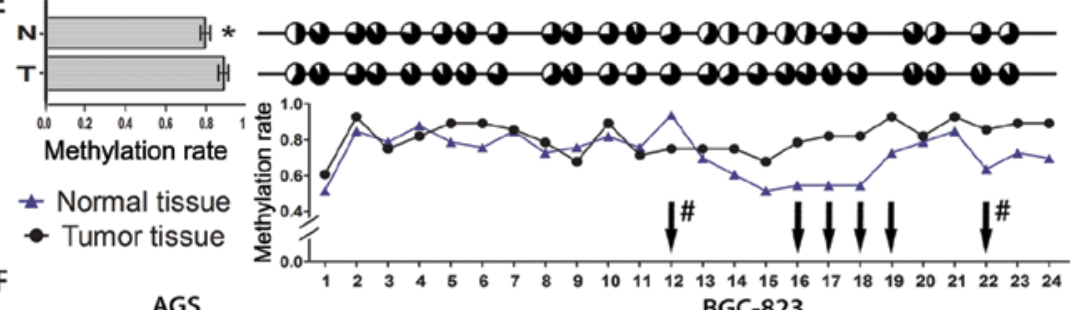

F AGS

Control

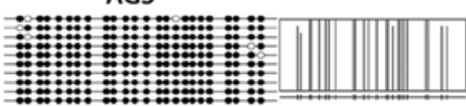

$2 \mu \mathrm{M}$

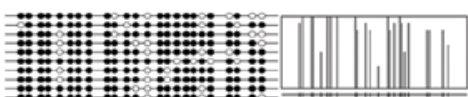

$10 \mu \mathrm{M}$

:

:

MGC-803

Control

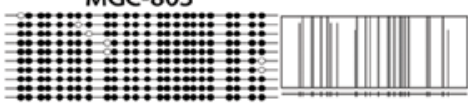

$2 \mu \mathrm{M}$

$10 \mu \mathrm{M}$

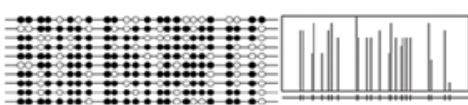

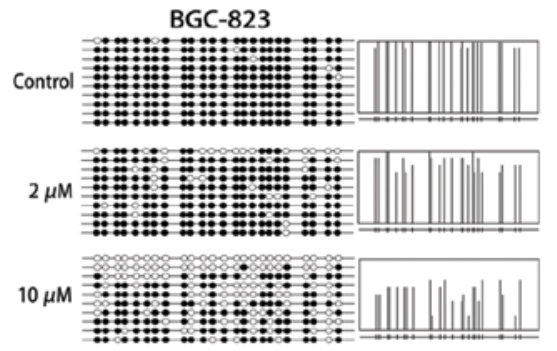

SGC-7901

Control

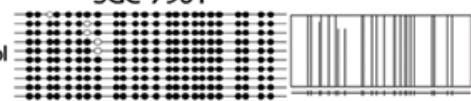

$2 \mu \mathrm{M}$

:

:
$10 \mu \mathrm{M}$

Figure 1. Evidence on methylation information of SEMA3B gene. (A) Chromosomal localization and structural organization of SEMA3B gene, containing exons 1-18. (B) CpG island and bisulfite PCR primer localization of SEMA3B gene determined by MethPrimer online software. Arrows 1-4 indicate CpG island region 1-4. (C) Detailed target DNA sequences within bisulfite PCR primer (F/R). CpG dinucleotides are underlined. (D) CpG demethylation rate of each gastric cell line. ${ }^{* \#}$ Compared to the control group $(\mathrm{P}<0.05)$. (E) Methylation level of normal and cancer tissues. Bar graph denotes total methylation rate of normal and cancer tissues. N, normal tissue; T, tumor tissue. White part in circle indicates unmethylated status. Black part in circle indicates methylated status. Line chart shows methylation rate of each $\mathrm{CpG}$ site. Arrows represent site of significant difference between tumor and normal tissues. ${ }^{\#} \mathrm{P}$-value determined by Fisher's exact test. (F) Detailed methylation status of each $\mathrm{CpG}$ site of individual clone. Open circle indicates unmethylated CpG site. Black circle indicates methylated $\mathrm{CpG}$ site. Bar chart sideward indicates methylation rate of each $\mathrm{CpG}$ site.

tests, and the differences were considered statistically significant when the P-value was $\leq 0.05$.

\section{Results}

Prediction of CPG islands based on SEMA3B gene DNA sequence. The SEMA3B gene is located in 3p21.3 of human chromosome with exons length of $\sim 3.4 \mathrm{~kb}$, coding 749 amino acids. Using MethPrimer online software (21), 4 CpG island regions were identified based on SEMA3B gene sequence (NCBI Reference Sequence: NM_004636). Conditions on CpG island identification had been set as default terms that island size $>100$, GC Percent $>50.0$ and Obs/Exp >0.6. Bisulfite PCR primer in island 3 was adopted in our research, including 24 $\mathrm{CpG}$ dinucleotides in the 5 ' region from exon 18 of SEMA3B gene (Fig. 1A-C). 

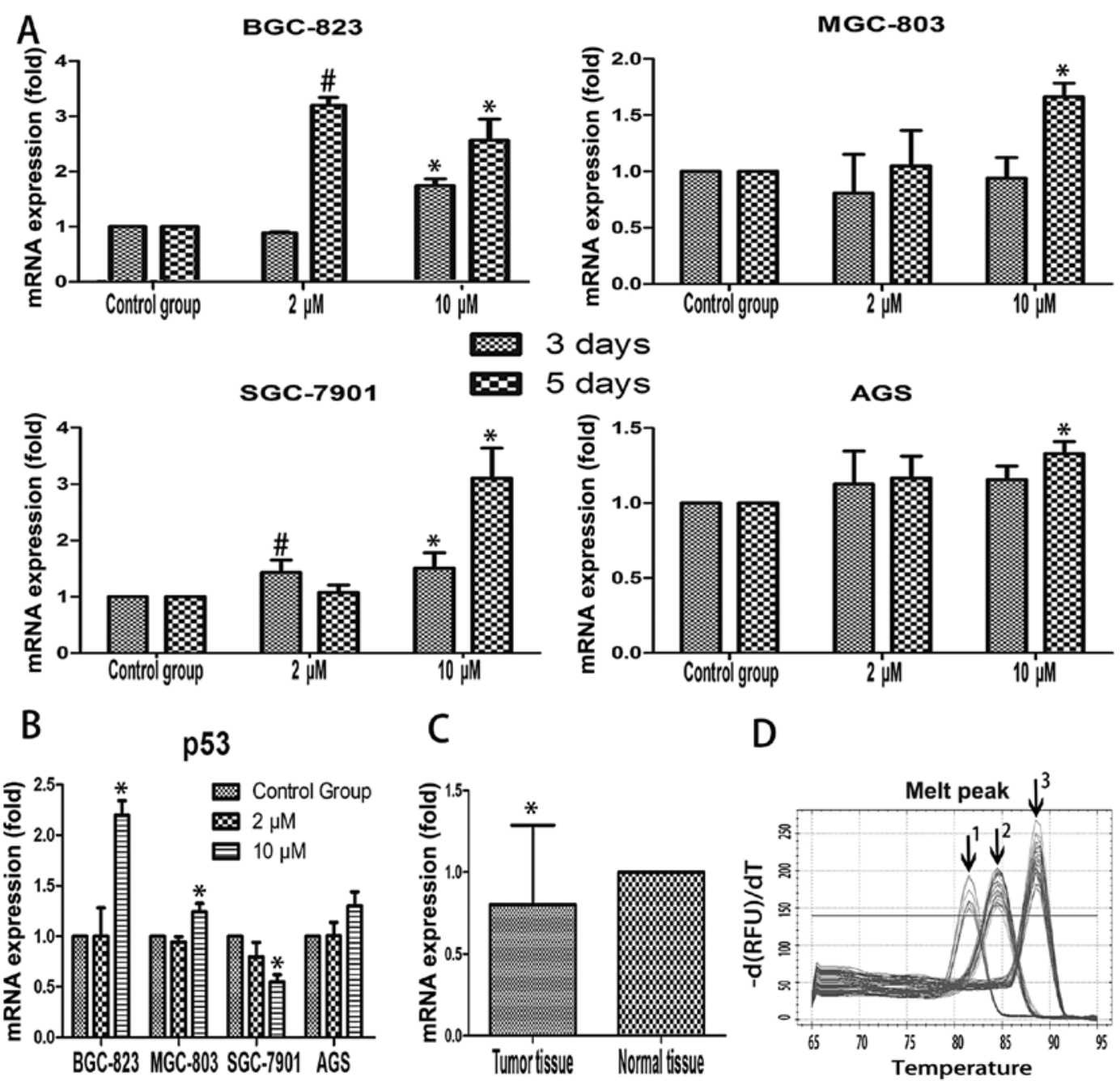

Figure 2. SEMA3B mRNA expression level analysis. (A) SEMA3B mRNA expression level in 4 gastric cell lines before and after the treatment of 5-Aza-2'deoxycytidine. Expression levels were calculated by quantitative real-time PCR analysis. GAPDH gene was used as internal reference gene and the $\Delta \Delta C T$ method was used for relative quantification. ${ }^{* * \#}$ Compared to the control group $(\mathrm{P}<0.05)$. (B) p53 mRNA expression level in 4 gastric cell lines before and after the treatment of 5-Aza-2'-deoxycytidine. "Compared to the control group $(\mathrm{P}<0.05)$. (C) SEMA3B mRNA level was analyzed in 30 gastric cancer tissues and corresponding normal gastric mucosa specimens. The bars represent standard deviations. Two-tailed Student's t-test was adopted to compare the SEMA3B expression levels. The expression was significantly lower in gastric cancer tissues compared to normal gastric mucosa specimens ( $\mathrm{P}=0.034)$. " Compared to the control group $(\mathrm{P}<0.05)$. (D) Melt curve of individual genes. Arrow 1, p53; Arrow 2, GAPDH; Arrow 3, SEMA3B.

SEMA3B expression is downregulated in GC tissues. Results of qRT-PCR showed 63\% of SEMA3B mRNA level was downregulated in primary $\mathrm{GC}$ samples, compared to the matched normal samples (19/30, P=0.034; Fig. 2C). Correlation analysis identified that SEMA3B mRNA expression is negatively correlated with tumor size and tumor $\mathrm{N}$-stage (Table II). We noted that advanced $\mathrm{N}$ stage showed less SEMA3B mRNA expression pattern $(\mathrm{P}<0.01)$.

5-Aza-2'-deoxycytidine restores SEMA3B gene expression based on different time and concentration patterns. Expression of SEMA3B gene was evaluated by qRT-PCR and western blotting, both of which were detected in $4 \mathrm{GC}$ cell lines (BGC-823, MGC-803, SGC-7901 and AGS). In 3-day treatment, compared to the control group, only BGC-823 and SGC-7901 showed increased mRNA expression following the dose cumulation of 5-Aza-2'-deoxycytidine $(\mathrm{P}<0.05)$. In 5-day treatment, all the gastric cell lines showed the tendency of increased mRNA expression at a concentration of $10 \mu \mathrm{M}$ of 5-Aza-2'-deoxycytidine except for BGC-823 ( $\mathrm{P}<0.05$; Fig. 2A). We further explored the Sema3b protein expression in 5-day treatment. BGC-823 and MGC-803 showed a significant increased Sema3b protein expression when dosage of 5-Aza-2'-deoxycytidine was elevated $(\mathrm{P}<0.05)$. This effect was only identified in SGC-7901 when it was compared with the control group at the $10 \mu \mathrm{M}$ concentration. AGS showed the opposite expression pattern when the concentration of 5-Aza-2'-deoxycytidine was up to $10 \mu \mathrm{M}$, the Sema3b protein expression was $<2 \mu \mathrm{M}$ (Fig. 3).

Elevated 53 expression is associated with SEMA3B re-expression. In order to explore the role of p53 on SEMA3B mRNA expression, we examined the p53 mRNA level by qRT-PCR, also with the concentration of 5-Aza-2'-deoxycytidine at 0,2 and $10 \mu \mathrm{M}$ (Fig. 2B). Results showed that p53 mRNA expression level was elevated in BGC-823, MGC-803 and AGS, compared to the control group. However, AGS failed to reach statistical significance $(\mathrm{P}>0.05)$. SGC-7901 showed 
Table II. Clinicopathological correlation of SEMA3B mRNA expression status.

\begin{tabular}{|c|c|c|c|c|}
\hline \multirow[b]{2}{*}{ Clinical characteristics } & \multicolumn{3}{|c|}{ SEMA3B mRNA expression status $(\mathrm{N})$} & \multirow{2}{*}{$\begin{array}{c}\begin{array}{c}\text { Correlation } \\
\text { analysis }\end{array} \\
\text { r, P-value }\end{array}$} \\
\hline & Upregulation & Downregulation & P-value ${ }^{a}$ & \\
\hline Gender & & & & $-0.017,0.927$ \\
\hline Male & 8 & 14 & $0.383^{\mathrm{b}}$ & \\
\hline Female & 3 & 5 & & \\
\hline Age (mean $\pm \mathrm{SD}$, years) & $66.73 \pm 11.4(11)$ & $63.47 \pm 7.6(19)$ & 0.355 & $0.076,0.689$ \\
\hline Tumor size $($ mean $\pm \mathrm{SD}, \mathrm{cm})$ & $3.73 \pm 1.3(11)$ & $4.95 \pm 2.7(19)$ & 0.063 & $-0.386, \mathbf{0 . 0 3 5}^{\mathrm{c}}$ \\
\hline Infiltration depth & & & & $0.043,0.821$ \\
\hline Submucosa & 1 & 4 & $0.529^{\mathrm{b}}$ & \\
\hline Lamina propria & 0 & 1 & & \\
\hline Muscular layer & 0 & 1 & & \\
\hline Serosa & 10 & 13 & & \\
\hline Lymphatic metastasis & & & & $-0.063,0.741$ \\
\hline Yes & 8 & 13 & $0.804^{\mathrm{b}}$ & \\
\hline No & 3 & 6 & & \\
\hline Borrmann type & & & & $0.017,0.928$ \\
\hline $\mathrm{I}+\mathrm{II}$ & 10 & 17 & $0.321^{\mathrm{b}}$ & \\
\hline III + IV & 1 & 2 & & \\
\hline Differentiation & & & & $-0.172,0.365$ \\
\hline Low & 9 & 11 & $0.246^{\mathrm{b}}$ & \\
\hline Medium-high & 2 & 8 & & \\
\hline T stage & & & & $0.023,0.905$ \\
\hline $\mathrm{T} 1-\mathrm{T} 2$ & 1 & 3 & $0.570^{\mathrm{b}}$ & \\
\hline T3 & 5 & 11 & & \\
\hline $\mathrm{T} 4$ & 5 & 5 & & \\
\hline $\mathrm{N}$ stage & & & & $-0.491, \mathbf{0 . 0 0 6}^{\mathrm{d}}$ \\
\hline N0-N1 & 9 & 6 & $\mathbf{0 . 0 0 3}^{\mathrm{b}}$ & \\
\hline $\mathrm{N} 2-\mathrm{N} 3$ & 2 & 13 & & \\
\hline Nerve tract involved & & & & $0.14,0.461$ \\
\hline Yes & 5 & 6 & 0.447 & \\
\hline No & 6 & 13 & & \\
\hline Vessel involved & & & & $-0.023,0.905$ \\
\hline Yes & 2 & 2 & $0.611^{\mathrm{b}}$ & \\
\hline No & 9 & 17 & & \\
\hline
\end{tabular}

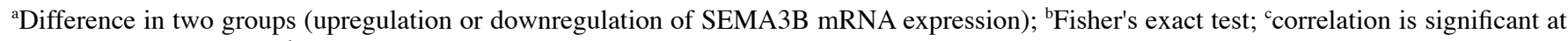
the 0.05 level (2-tailed); ${ }^{\mathrm{d}}$ correlation is significant at the 0.01 level (2-tailed).

decreased p53 mRNA expression level in $10 \mu \mathrm{M}$ of 5-Aza-2'deoxycytidine compared to the control group $(\mathrm{P}<0.05)$. By searching available databases, Agilent Literature Search tool finds publications related to search terms and creates interaction networks based on the search result. We used the key words 'Sema3b, p53, neuropilins, vascular endothelial growth factor, cancer' and 20 articles were found. A clear relationship was shown in the generated network, in which vegfa and tp53 exchanged information with outside more frequently and $\mathrm{mmp} 2$ was an important intermediary agent connecting semaphoring with vegfa (Fig. 4).
Methylation status of SEMA3B gene exon 18 affects transcript activities of $S E M A 3 B$. Bisulfite sequencing is regarded as the gold standard to analyze the methylation status of one target sequence. We performed bisulfite sequencing on gastric cell lines and tissues of tumor and normal gastric mucosa. Nearly $100 \%$ of CpGs in exon 18 of SEMA3B showed methylated status in all 4 gastric cell lines. After treatment of 5-Aza-2'deoxycytidine for 5 days with the concentration at 2 and $10 \mu \mathrm{M}, \mathrm{CpG}$ demethylation rate improved significantly in all 4 gastric cell lines, compared to the control group $(\mathrm{P}<0.05$; Fig. 1D and F). Furthermore, we detected the methylation 


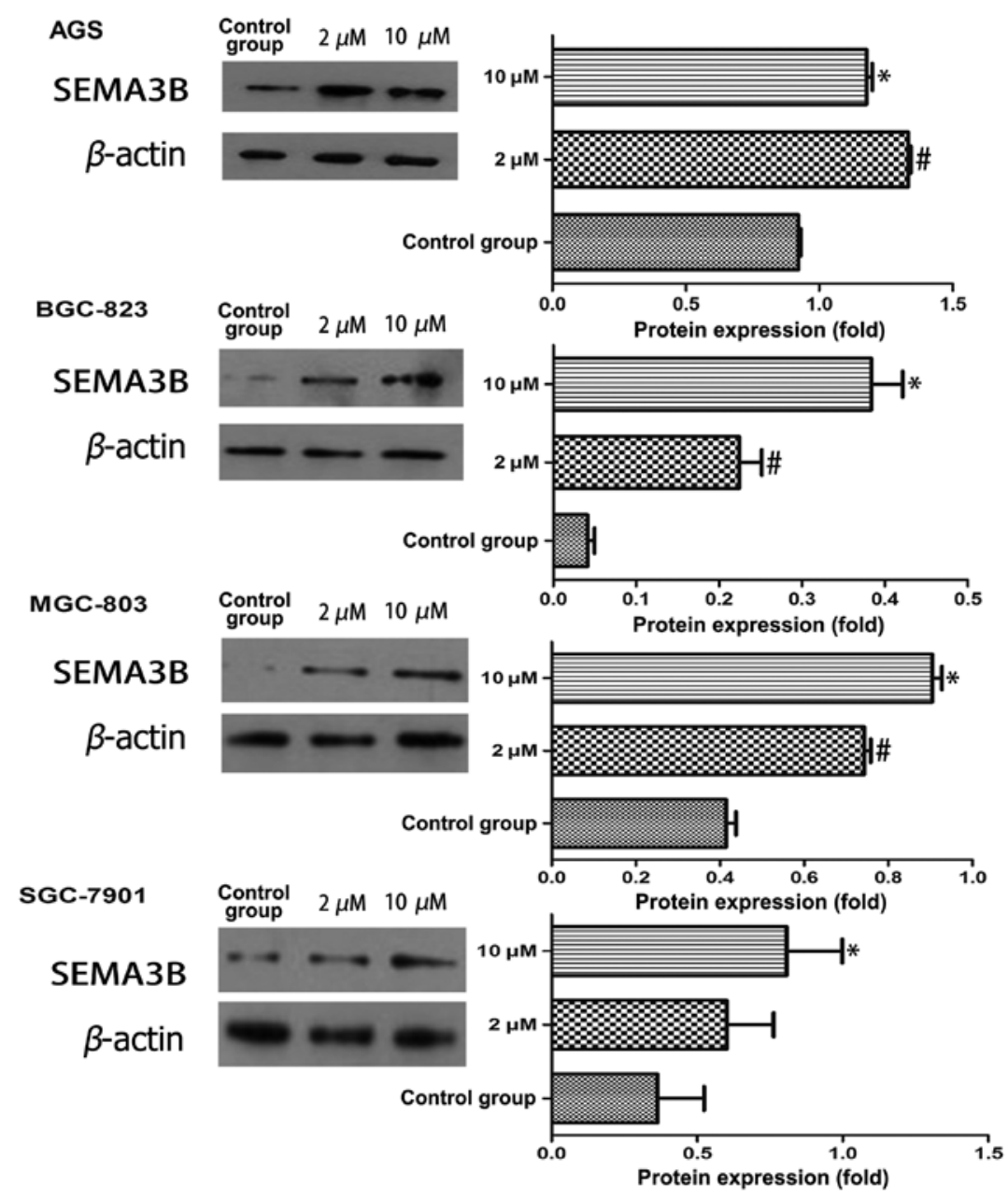

Figure 3. Western blot analysis of Sema3b protein in gastric cell lines. ${ }^{*}{ }^{*}$ Compared to the control group $(\mathrm{P}<0.05)$.

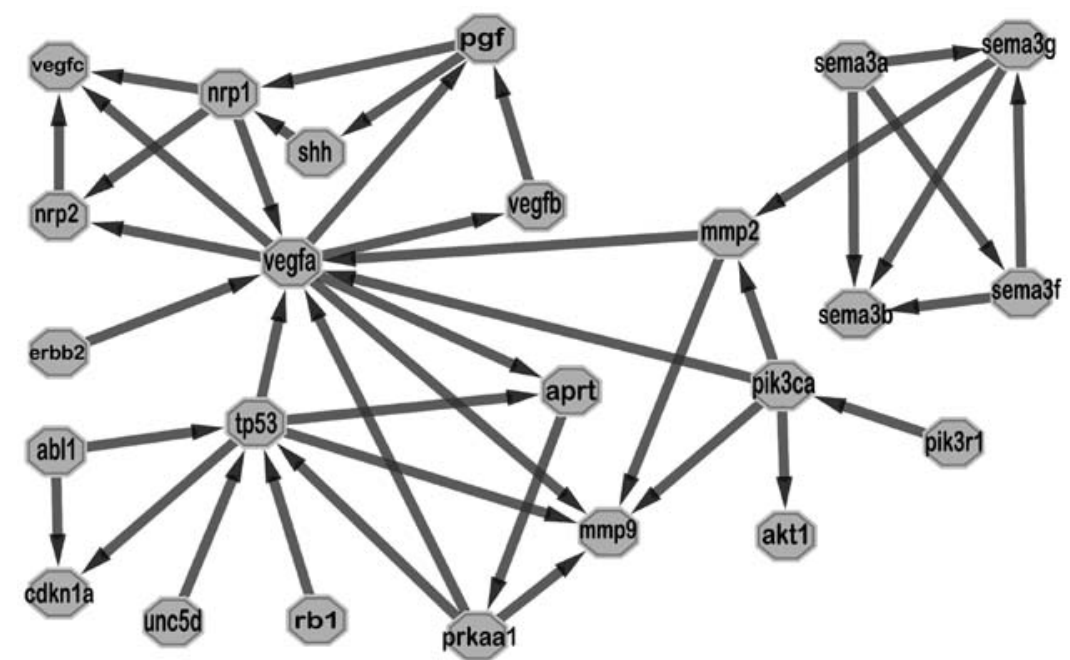

Figure 4. Relationship between SEMA3B and its correlated regulators. Data were analyzed by Agilent Literature Search Software; run on the platform of Cytoscape. Visual style, Bingo style. Nodes, 24. Edge, 41.

levels of the exon 18 of SEMA3B, compared among the available 15 paired tissues of tumor and normal gastric mucosa (Fig. 1E). It was observed that the methylation level was slightly but significantly higher in tumor tissue compared to normal gastric mucosa $(\mathrm{P}<0.05)$. Regarding the methylation level of each paired $\mathrm{CpG}$ island which had different effect on methylation status in cancer, CpG island -16, -17, -18, -19, -22 in the tumor group showed hypermethylated status $(\mathrm{P}<0.05)$, while $\mathrm{CpG}$ island -12 in paired normal tissue group showed hypermethylated status $(\mathrm{P}<0.05)$. 


\section{Discussion}

The present study was conducted to determine the role of SEMA3B in GC with regard to the relationship between methylation status and gene expression pattern.

Quantitative RT-PCR was used to assess mRNA level of SEMA3B gene in primary gastric tumor and cell lines (BGC-823, SGC-7901, MGC-803 and AGS). Data showed that the SEMA3B gene was downregulated in gastric tissue compared to normal gastric mucosa tissue. This suggested a decrease in SEMA3B expression may be involved in the development of GC. Correlation analysis between mRNA expression of SEMA3B and clinicopathological parameters revealed that SEMA3B was involved in tumor growth and also affected regional lymph node infiltration. As a type of secreted semaphorins, Sema3s bind to a holoreceptor complex that consists of Nrps as ligand binding. With the exception of binding to Sema3s, Nrps could serve as VEGF family protein co-receptors (22). It was suggested that Sema3s compete for the binding to Nrp1 (neuropilins) with VEGF to inhibit VEGF-induced angiogenesis (23). Lymph node infiltration was related to lymphatic vasculature building, which was attributed to signaling by VEGF-C and regulated by Nrp2 (24). This suggested that Sema3s could also possibly function as anti-lymphangiogenic signaling molecules through the binding affinity to Nrp2. Previous studies showed that Sema3s are also involved in the lymph node metastasis of prostate cancer, and were likely to modulate the behavior of prostate cancer with an antitumor effect (25). In the present study, with the deficiency of SEMA3B mRNA expression, its anti-angiogenic and anti-lymphangiogenic effect was too low to induce tumor growth, and lymph node infiltration was more common in GC. According to earlier studies, SEMA3B mRNA level was also observed to be downregulated in different types of tumors. Pronina et al (26) reported that SEMA3B expression levels were frequently decreased in primary tumor extracts of kidney, lung, breast, ovarian and colorectal cancer. Furthermore, this phenomenon was confirmed in cell lines of breast cancer, epithelial ovarian carcinoma and renal cell carcinoma.

We further explored the potential influencing factors that affect the expression level of SEMA3B, in which methylation status of SEMA3B in gastric tissue was detected. Analysis of exon 18 of SEMA3B indicated that tissues from gastric tumor showed higher methylated status than normal tissues $(\mathrm{P}<0.05)$, in which methylation in $\mathrm{CpG}$ site $-16,-17,-18,-19$, -22 weighted heavily. Epigenetic alteration in SEMA3B DNA sequence is regarded as an important gene expression silencing pathway demonstrated in several types of tumor, such as that in gallbladder carcinoma (27). Kuroki et al (28) reported that SEMA3B hypermethylation is responsible for silencing SEMA3B expression in NSCLC cell line by methylationspecific PCR. However, this result was not in accordance with that in gliomas which showed SEMA3B was not frequently methylated (29). Different methylation rate has been reported in GC which varied from $20 \%$ (30) to $60 \%$ (31). In our cases, the methylation rate of SEMA3B in exon 18 from gastric tumor tissue was $88 \%$, significantly higher than the paired normal tissue $(79 \% ; \mathrm{P}<0.05)$.

5-Aza-2'-deoxycytidine has been used as a demethylation agent in recent years. After treatment with 5-Aza-2'-deoxycytidine, a demethylation state was detected in the 4 GC cell lines (BGC-823, MGC-803, SGC-7901 and AGS). Sema3b protein expression was significantly higher in the 5-Aza-2'deoxycytidine-treated group compared with that in the control group. qRT-PCR further confirmed that in 5-day treatment, 5-Aza-2'-deoxycytidine could come to the largest effect confronted with the hypermethylation status of SEMA3B in gastric cell lines. Meanwhile, p53 expression was detected following the impact of 5-Aza-2'-deoxycytidine. Except for SGC-7901, all remaining gastric cell lines showed higher p53 mRNA expression after the treatment of 5-Aza-2'deoxycytidine at the concentration of $10 \mu \mathrm{M}$. Ochi et al (13) demonstrated that introduction of exogenous p53 into a glioblastoma cell line which is lacking wild-type p53 markedly induced expression of SEMA3B mRNA. This result suggested that SEMA3B plays some role as a direct target of p53 and it was consistently comparable to our data. Notably, SGC-7901 showed the opposite outcome that remains to be demonstrated in future studies, which indicated a more complex mechanism that had an effect on the relationship between p53 and SEMA3B in different genetic backgrounds. Network analysis related to sema3b, VEGF, neuropilins and p53 showed complex pathways between them, in which mmp2 seemed to be an important channel joining sema3b with vegfa. As an index of tumor invasion ability, mmp2 has been shown to be inhibited by SEMA3G (32).

In summary, we confirmed that the methylation status of the SEMA3B gene in gastric tumor was significantly higher than that in paired normal gastric mucosa. We showed the epigenetic inactivation of SEMA3B in several types of GC cell lines and 5-Aza-2'-deoxycytidine could reverse the hypermethylation status of SEMA3B, which may benefit future studies exploring the application of demethylating agents in clinical usage for GC.

\section{References}

1. Parkin DM, Bray F, Ferlay J and Pisani P: Global cancer statistics, 2002. CA Cancer J Clin 55: 74-108, 2005.

2. Rivera F, Vega-Villegas ME and Lopez-Brea MF: Chemotherapy of advanced gastric cancer. Cancer Treat Rev 33: 315-324, 2007.

3. Yazdani U and Terman JR: The semaphorins. Genome Biol 7: 211, 2006.

4. Chilton JK and Guthrie S: Cranial expression of class 3 secreted semaphorins and their neuropilin receptors. Dev Dyn 228: 726-733, 2003.

5. Giger RJ, Cloutier JF, Sahay A, et al: Neuropilin-2 is required in vivo for selective axon guidance responses to secreted semaphorins. Neuron 25: 29-41, 2000.

6. Falk J, Bechara A, Fiore R, et al: Dual functional activity of semaphorin $3 \mathrm{~B}$ is required for positioning the anterior commissure. Neuron 48: 63-75, 2005.

7. Nawabi H, Briancon-Marjollet A, Clark C, et al: A midline switch of receptor processing regulates commissural axon guidance in vertebrates. Genes Dev 24: 396-410, 2010.

8. Tamagnone L and Comoglio PM: To move or not to move? Semaphorin signalling in cell migration. EMBO Rep 5: 356-361, 2004.

9. Sekido Y, Bader S, Latif F, et al: Human semaphorins $\mathrm{A}(\mathrm{V})$ and IV reside in the 3 p21.3 small cell lung cancer deletion region and demonstrate distinct expression patterns. Proc Natl Acad Sci USA 93: 4120-4125, 1996.

10. Tomizawa Y, Sekido Y, Kondo M, et al: Inhibition of lung cancer cell growth and induction of apoptosis after reexpression of 3 p21.3 candidate tumor suppressor gene SEMA3B. Proc Nat Acad Sci USA 98: 13954-13959, 2001.

11. Tse C, Xiang RH, Bracht T and Naylor SL: Human Semaphorin 3B (SEMA3B) located at chromosome 3p21.3 suppresses tumor formation in an adenocarcinoma cell line. Cancer Res 62: $542-546,2002$ 
12. Tischoff I, Markwarth A, Witzigmann H, et al: Allele loss and epigenetic inactivation of $3 \mathrm{p} 21.3$ in malignant liver tumors. Int J Cancer 115: 684-689, 2005.

13. Ochi K, Mori T, Toyama Y, Nakamura Y and Arakawa H: Identification of semaphorin3B as a direct target of p53. Neoplasia 4: 82-87, 2002.

14. Castro-Rivera E, Ran S, Brekken RA and Minna JD: Semaphorin 3B inhibits the phosphatidylinositol 3-kinase/Akt pathway through neuropilin-1 in lung and breast cancer cells. Cancer Res 68: 8295-8303, 2008.

15. Castro-Rivera E, Ran S, Thorpe P and Minna JD: Semaphorin 3B (SEMA3B) induces apoptosis in lung and breast cancer, whereas VEGF165 antagonizes this effect. Proc Natl Acad Sci USA 101: 11432-11437, 2004.

16. Koyama N, Zhang J, Huqun, et al: Identification of IGFBP-6 as an effector of the tumor suppressor activity of SEMA3B. Oncogene 27: 6581-6589, 2008.

17. Varshavsky A, Kessler O, Abramovitch S, et al: Semaphorin-3B is an angiogenesis inhibitor that is inactivated by furin-like proprotein convertases. Cancer Res 68: 6922-6931, 2008.

18. Rolny C, Capparuccia L, Casazza A, et al: The tumor suppressor semaphorin $3 \mathrm{~B}$ triggers a prometastatic program mediated by interleukin 8 and the tumor microenvironment. J Exp Med 205: 1155-1171, 2008.

19. Livak KJ and Schmittgen TD: Analysis of relative gene expression data using real-time quantitative PCR and the 2(-Delta Delta $\mathrm{C}(\mathrm{T})$ ) method. Methods 25: 402-408, 2001.

20. Vailaya A, Bluvas P, Kincaid R, Kuchinsky A, Creech M and Adler A: An architecture for biological information extraction and representation. Bioinformatics 21: 430-438, 2005.

21. Li LC and Dahiya R: MethPrimer: designing primers for methylation PCRs. Bioinformatics 18: 1427-1431, 2002.

22. Gluzman-Poltorak Z, Cohen T, Herzog Y and Neufeld G: Neuropilin-2 is a receptor for the vascular endothelial growth factor (VEGF) forms VEGF-145 and VEGF-165. J Biol Chem 275: 29922, 2000.
23. Soker S, Miao HQ, Nomi M, Takashima S and Klagsbrun M: VEGF165 mediates formation of complexes containing VEGFR-2 and neuropilin-1 that enhance VEGF165-receptor binding. J Cell Biochem 85: 357-368, 2002.

24. Haiko P, Makinen T, Keskitalo S, et al: Deletion of vascular endothelial growth factor C (VEGF-C) and VEGF-D is not equivalent to VEGF receptor 3 deletion in mouse embryos. Mol Cell Biol 28: 4843-4850, 2008.

25. Li K, Chen MK, Li LY, et al: The predictive value of semaphorins 3 expression in biopsies for biochemical recurrence of patients with low- and intermediate-risk prostate cancer. Neoplasma 60: 683-689, 2013.

26. Pronina IV, Loginov VI, Prasolov VS, et al: Alteration of SEMA3B gene expression levels in epithelial tumors. Mol Biol (Mosk) 43: 439-445, 2009 (In Russian).

27. Riquelme E, Tang M, Baez S, et al: Frequent epigenetic inactivation of chromosome $3 \mathrm{p}$ candidate tumor suppressor genes in gallbladder carcinoma. Cancer Lett 250: 100-106, 2007.

28. Kuroki T, Trapasso F, Yendamuri S, et al: Allelic loss on chromosome 3p21.3 and promoter hypermethylation of semaphorin 3B in non-small cell lung cancer. Cancer Res 63: 3352-3355, 2003.

29. Hesson L, Bieche I, Krex D, et al: Frequent epigenetic inactivation of RASSF1A and $B L U$ genes located within the critical 3 p21.3 region in gliomas. Oncogene 23: 2408-2419, 2004.

30. Bernal C, Vargas M, Ossandon F, et al: DNA methylation profile in diffuse type gastric cancer: evidence for hypermethylation of the BRCA1 promoter region in early-onset gastric carcinogenesis. Biol Res 41: 303-315, 2008

31. Bernal C, Aguayo F, Villarroel C, et al: Reprimo as a potential biomarker for early detection in gastric cancer. Clin Cancer Res 14: 6264-6269, 2008.

32. Zhou X, Ma L, Li J, Gu J, Shi Q and Yu R: Effects of SEMA3G on migration and invasion of glioma cells. Oncol Rep 28: 269-275, 2012. 